\title{
2 Research Square \\ Prediction of higher thermoelectric performance in BiOCuSe by weakening electron-polar optical phonon scattering
}

\section{Tianqi Zhao}

University College London

\section{Quinn Gibson}

Princeton University

\section{Luke Daniels}

University of Liverpool https://orcid.org/0000-0002-7077-6125

\section{Ben Slater}

University College London https://orcid.org/0000-0001-9738-5848

Furio Cora ( $\nabla$ f.cora@ucl.ac.uk)

University College London

\section{Article}

Keywords: BiOCuSe, ZT, electron-phonon scattering, thermoelectric performance, carrier mobility

Posted Date: July 10th, 2020

DOl: https://doi.org/10.21203/rs.3.rs-37817/v1

License: (c) (1) This work is licensed under a Creative Commons Attribution 4.0 International License. Read Full License 


\section{Abstract}

BiOCuSe is a promising thermoelectric material, but its applications are hindered by low carrier mobility. We use first principles calculations to analyse electron-phonon scattering mechanisms and evaluate their contributions to the thermoelectric figure of merit $Z T$. The combined scattering of carriers by polar optical (PO) and longitudinal acoustic (LA) phonons yields an intrinsic hole mobility of $32 \mathrm{~cm}^{2} \mathrm{~V}^{-1} \mathrm{~s}^{-1}$ at room temperature and a temperature power law of $T^{-1.5}$, which agree well with experiments. We demonstrate that electron phonon scattering in the $\mathrm{Cu}$-Se layer dominates at low $T$, while contributions from the Bi-O layer become increasingly significant at higher $T$. At room temperature, $Z T$ is calculated to be 0.48 and can be improved by $30 \%$ through weakening PO phonon scattering in the Cu-Se layer. This finding agrees with the experimental observation that weakening the carrier-phonon interaction by Te substitution in the Cu-Se layer improves mobility and $Z T$. At high $T$, the figure of merit is improved by weakening phonon scattering in the Bi-O layer instead. The theoretical $Z T$ limit of BiOCuSe is calculated to be 2.5 at $875 \mathrm{~K}$.

\section{Introduction}

Thermoelectric materials are attracting increasing attention in applications such as waste heat recovery and solid-state cooling. ${ }^{1-3}$ The thermoelectric performance of materials is evaluated by the dimensionless thermoelectric figure of merit $Z T$, which is determined by the interplay of Seebeck coefficient $S$, electrical conductivity $s$, thermal conductivity $k$, and absolute temperature $T$ through the equation $Z T=S^{2} s T / k$. The combined quantity $S^{2} s$ is called the thermoelectric power factor, and $k$ is composed of the electronic thermal conductivity $k_{\mathrm{e}}$ and lattice thermal conductivity $k_{L}$, i.e. $k=k_{\mathrm{e}}+k_{L}$. Oxides were once believed to be poor thermoelectric materials due to their high thermal conductivity and low carrier mobility. ${ }^{3}$ However, practical high temperature thermoelectric applications call for oxides because of their high thermal and chemical stability. Currently, several families of promising thermoelectric oxides have been discovered, ${ }^{3-5}$ among which $\mathrm{Na}_{\mathrm{x}} \mathrm{CoO}_{2}$ and $\mathrm{SrTiO}_{3}$ based materials possess relatively high thermoelectric power factors. ${ }^{6,7}$ Mixed anion compounds, such as the layered BiOCuSe material which combines characteristics of both oxide and chalcogenide materials, are advantageous in thermoelectric applications because of their intrinsically low thermal conductivity $(\sim 1$ $\mathrm{W} \mathrm{m}^{-1} \mathrm{~K}^{-1}$ at room temperature) and high thermal stability. ${ }^{8,9}$ BiOCuSe-based materials have emerged as state-of-the-art thermoelectric oxychalcogenides since 2010.9,10 Various doping strategies, such as substitution of Bi with alkali, ${ }^{11,12}$ alkaline earth, ${ }^{9,13-16}$ and post-transition metals such as lead, ${ }^{17-20}$ have been successfully demonstrated to obtain $Z T$ values close or beyond unity at high temperatures. The highest p-type $Z T$ have reached 1.5 at $873 \mathrm{~K}$ by employing a dual-doping method at the Bi site in $\mathrm{Bi}_{0.88} \mathrm{Ca}_{0.06} \mathrm{~Pb}_{0.06} \mathrm{OCuSe}^{21}$ However, the low carrier mobility of BiOCuSe $\left(\sim 20 \mathrm{~cm}^{2} \mathrm{~V}^{-1} \mathrm{~s}^{-1}\right.$ at room temperature vs. $525 \mathrm{~cm}^{2} \mathrm{~V}^{-1} \mathrm{~s}^{-1}$ of $\mathrm{Bi}_{2} \mathrm{Te}_{3}$ at $300 \mathrm{~K}$ ) limits further optimization of the thermoelectric performance. ${ }^{1,20,22}$ 
Computational analysis that applies deformation potential (DP) theory has been used to explain the charge transport properties of BiOCuSe by assuming that longitudinal acoustic (LA) phonons dominates charge transport, ${ }^{23-25}$ which is based on the fact that the measured mobility obeys the temperature power law of $T^{1.5} .{ }^{15}$ This power law assumes the existence of a single parabolic band (SPB). ${ }^{22,24}$ However, there is an eight-fold valley degeneracy near the valence band maxima of BiOCuSe as revealed in previous work, ${ }^{23}$ so the assumption of SPB in BiOCuSe is debatable. Electron-polar optical (PO) phonon scattering, which accounts for the interaction between charge carriers and the electric field generated by vibrations of atoms with opposite charge, is important for polar semiconductors, such as $\mathrm{SrTiO}_{3}$ and $\mathrm{CH}_{3} \mathrm{NH}_{3} \mathrm{Pbl}_{3},{ }^{26-28}$ because of the large electronegativity difference between elements. The effect of PO phonon scattering has been ignored in BiOCuSe to the best of our knowledge. Here, by performing first-principles calculations we demonstrate that PO phonon scattering plays a more fundamental role than LA phonon scattering in the charge transport properties of BiOCuSe, particularly at low temperature ( $<500 \mathrm{~K}$ ). Our results highlight that contributions from the $\mathrm{Cu}-\mathrm{Se}$ and $\mathrm{Bi}-\mathrm{O}$ layers separately dominate in different temperature ranges and carrier concentrations, and thus suggest ways for a rational optimization of the thermoelectric performance of BiOCuSe.

\section{Results}

BiOCuSe is formed by ionic layers of composition $\left[\mathrm{Bi}_{2} \mathrm{O}_{2}\right]^{2+}$ and $\left[\mathrm{Cu}_{2} \mathrm{Se}_{2}\right]^{2-}$, and adopts a tetragonal cell with space group $P 4 / n m m$ (Fig. 1). The optimized lattice constants in our calculations $(a=3.926 \AA, c=$ $8.957 \AA$ ) show good agreement with experimental values $(a=3.930 \AA, c=8.927 \AA) .{ }^{9}$ The electronic band structures and density of states (DOS) calculated with the PBE $+U+S O C$ functional are shown in Fig. 1 a. The calculated band gap is $0.37 \mathrm{eV}$. Previous HSE + SOC calculations show a band gap of $0.81 \mathrm{eV}$, which is closer to the experimental measure of $0.80 \mathrm{eV} .^{9,23}$ However, our band structure compares well with that calculated with the more expensive HSE + SOC functional after a rigid band shift of $0.44 \mathrm{eV}^{23,29}$ The top of the valence band of BiOCuSe is composed of Se and Cu orbitals and the bottom of the conduction band is constituted of Bi orbitals. The valence band maximum (VBM) is located along the $\mathrm{M}-\Gamma$ direction. The charge density at the VBM is shown in Fig. 1c. There are three valence band maxima, one along $\Gamma-M$, one at $Z$, and one along $Z-R$ with only a minor energy difference ( $15 \mathrm{meV}$ between VBM and the valence band maxima at Z, $51 \mathrm{meV}$ between VBM and the valence band maxima along Z-R). The hole effective mass around the three valence band maxima is calculated to be $0.21 \mathrm{~m}_{0}, 0.68 \mathrm{~m}_{0}$, and $4.60 \mathrm{~m}_{0}$, (where $m_{0}$ is the electron mass), respectively, which agree with previous theoretical results ( $Г-\mathrm{M}: 0.23, \mathrm{Z}-\Gamma: 0.47$, and R-Z: 4.53$){ }^{23}$

The phonon band structure and phonon DOS of BiOCuSe are shown in Fig. 1b. The phonons with frequencies above $200 \mathrm{~cm}^{-1}$ are mainly projected to oxygen and those below $200 \mathrm{~cm}^{-1}$ are mostly due to the heavier atoms. The $\Gamma$ point has $D_{4 h}$ point group symmetry, with phonons belonging to the following irreducible representations: $\Gamma=4 \mathrm{~A}_{2 \mathrm{u}}+2 \mathrm{~A}_{1 \mathrm{~g}}+2 \mathrm{~B}_{1 \mathrm{~g}}+4 \mathrm{E}_{\mathrm{u}}+4 \mathrm{E}_{\mathrm{g}}$. For the doubly degenerate $\mathrm{E}_{\mathrm{u}}$ and $\mathrm{E}_{\mathrm{g}}$ modes, atoms vibrate in plane, while for $A_{2 u}, A_{1 g}, B_{1 g}$, and $B_{2 u}$ modes, atoms vibrate out of plane. 
Figure 1e shows the six PO modes of BiOCuSe. The calculated frequencies of the PO modes compare well with literature (Table S1). ${ }^{30}$ The lower four PO phonon modes with frequencies below $200 \mathrm{~cm}^{-1}$ are mainly due to the atomic displacements in the Cu-Se layer, while the two PO modes with frequencies above $200 \mathrm{~cm}^{-1}$ are due to oxygen vibrations.

In order to discriminate the relative importance of LA and PO phonon scattering on charge transport in BiOCuSe, the LA phonon scattering is calculated by using deformation potential (DP) theory ${ }^{31}$ and the PO phonon scattering is calculated by accounting for the Fröhlich interaction. ${ }^{27}$ The DP constants are calculated by monitoring the variation of the energy of VBM with strain (Fig. 1d). Although this method is approximate $^{32}$ it has previously been successfully applied to describe the LA phonon scattering of $\mathrm{CH}_{3} \mathrm{NH}_{3} \mathrm{Pbl}_{3}$, and the calculated DP constants ( $4.3 \mathrm{eV}$ for electrons and $1.5 \mathrm{eV}$ for holes) ${ }^{33}$ are very close to experimental results (2.93 for electrons, $2.2 \mathrm{eV}$ for holes). ${ }^{34}$ The DP constants for holes in BiOCuSe are calculated to be 4.5 and $4.1 \mathrm{eV}$ in the $a$ and $c$ directions, respectively (Table 1 ). We see no reason why the DP constant of holes in BiOCuSe should be as high as $24 \mathrm{eV}^{23,24}$ except for fitting experimental results. The elastic constants of BiOCuSe are calculated to be 155.1 and $103.7 \mathrm{GPa}$ in a and c directions, respectively. The Young's modulus is 104.1 and $63.5 \mathrm{GPa}$ in the a and c directions, respectively, which agree well with the experimental value of $76.5 \mathrm{GPa}$ measured on polycrystalline samples. ${ }^{35}$ 
Table 1

Deformation potential $E_{1}$, elastic constant $C_{\mathrm{ii}}$, Young's Modulus, LA phonon scattering limited relaxation time $\tau_{\mathrm{LA}}$, and mobility $\mu_{\mathrm{LA}}, \mathrm{PO}$ phonon scattering limited relaxation time $\tau_{\mathrm{PO}}$ and mobility $\mu_{\mathrm{PO}}$, and the total carrier mobility limited by LA and PO phonon scattering $\mu_{\text {Total }}$ for holes in BiOCuSe at $300 \mathrm{~K}$.

\begin{tabular}{|c|c|c|c|}
\hline \multirow[b]{2}{*}{ direction } & \multicolumn{2}{|c|}{ This work } & \multirow[t]{2}{*}{ Experiment } \\
\hline & a & c & \\
\hline$E_{1}(\mathrm{eV})$ & 4.5 & 4.1 & \\
\hline$C_{i i}(\mathrm{GPa})$ & 155.1 & 103.7 & \\
\hline Young's Modulus (GPa) & 104.1 & 63.5 & $76.5^{35}$ \\
\hline$\tau_{\mathrm{LA}}(\mathrm{fs})$ & 80 & & \\
\hline$\tau_{\mathrm{PO}}(\mathrm{fs})$ & 15.0 & & \\
\hline$\mu_{\mathrm{LA}}\left(\mathrm{cm}^{2} \mathrm{~V}^{-1} \mathrm{~s}^{-1}\right)$ & 168 & 64.7 & \\
\hline$\mu_{\mathrm{PO}}\left(\mathrm{cm}^{2} \mathrm{~V}^{-1} \mathrm{~s}^{-1}\right)$ & 45.1 & 17.7 & \\
\hline$\mu_{\text {Total }}\left(\mathrm{cm}^{2} \mathrm{~V}^{-1} \mathrm{~s}^{-1}\right)$ & 31.6 & 12.4 & $20^{15}$ \\
\hline
\end{tabular}

Fig. $2 \mathrm{a}$ and $2 \mathrm{~b}$ show the temperature dependence of hole mobility calculated considering both LA and PO phonon scattering, and the limiting contributions when one only of the two terms is accounted for. The calculated room temperature mobilities in the $\boldsymbol{a}$ and $\boldsymbol{c}$ directions are $31.6 \mathrm{~cm}^{2} \mathrm{~V}^{-1} \mathrm{~s}^{-1}$ and $12.4 \mathrm{~cm}^{2} \mathrm{~V}^{-1} \mathrm{~s}^{-1}$, close to the experimental value $\left(\sim 20 \mathrm{~cm}^{2} \mathrm{~V}^{-1} \mathrm{~s}^{-1}\right)$ for pristine BiOCuSe measured on polycrystalline samples which is thus reported as isotropic. ${ }^{15}$ We attribute the mobility anisotropy between in-plane and out-of-plane directions to the anisotropy of effective mass ( $0.21 \mathrm{~m}_{0}$, in plane vs. $0.68 \mathrm{~m}_{0}$ out-of-plane), clearly linked with the layered structure of the compound. Our results indicate that the PO dominates over the LA phonon scattering for both in-plane and out-of-plane directions, in particular at low temperature; only for temperatures above $600 \mathrm{~K}$ does the LA scattering become the predominant mobility-limiting mechanism. The combined scattering of LA and PO phonons results in the power law of $T^{1.5}$ and $T^{1.6}$ in $\boldsymbol{a}$ and $\boldsymbol{c}$ directions respectively, in good agreement with the power low from experiment. ${ }^{15}$ The LA phonon scattering limited mobility (i.e. the mobility obtained neglecting PO scattering) follows a power law of $T$ 2.4. The assumption made until now when investigating mobility in BiOCuSe that LA phonon scattering follows a power law of $\sim T^{1.5,15,20,24}$ is therefore incorrect. This can be attributed to the multivalley valence bands of BiOCuSe, as occurs for InTe, where LA phonon scattering coupled with intervalley scattering results in a power law of $T^{2.01}{ }^{36} \mathrm{~A}$ valley degeneracy of eight is found in previous theoretical 
work and confirmed by our band structure calculations shown in Figure $1 .{ }^{23}$ In our analysis, the multiple valley scattering effects are included by calculating the scattering between electron states with the same energy in different valleys.

We further separated the effects of PO modes above and below $200 \mathrm{~cm}^{-1}$ to discriminate their relative contribution to hole mobility. As shown in Fig. $2 \mathrm{c}$ and $2 \mathrm{~d}$, the heavier atoms in the Cu-Se layer (modes below $200 \mathrm{~cm}^{-1}$ ) play a dominant role at $T$ below $\sim 500 \mathrm{~K}$, while oxygen vibrations become more important on increasing $T$ above $\sim 500 \mathrm{~K}$. It is interesting to note that increased carrier mobility has been reported experimentally upon Te doping into the Cu-Se layer, which weakens the electron-phonon scattering through higher bond covalency. ${ }^{22}$ A temperature power law of $T^{1.9}$ is obtained for the Te doped samples. ${ }^{22}$ This is consistent with our simulation, because when the polar optical phonon scattering from the Cu-Se layer is weakened, the power law will increase towards that of LA phonons.

Fig. 3 shows the dependence of the thermoelectric coefficients of BiOCuSe on carrier concentration at $300 \mathrm{~K}$ for the in-plane direction. For simplicity, we only discuss results in the in-plane direction in the following. Both LA and PO scattering mechanisms are included. The Seebeck coefficient $S$ is strongly affected by the band gap size due to the possible bipolar effect. Therefore, we applied a rigid band shift $(0.44 \mathrm{eV})$ on the conduction bands to match the gap of $0.81 \mathrm{eV}$ from experiment and HSE06+SOC calculations. Fig. $3 a$ shows that $S$ decreases with increasing carrier concentration and has excellent quantitative match with experimental results, especially at higher carrier concentrations. Fig. 3b and 3c show that both electrical conductivity $s$ and electronic thermal conductivity $k_{\mathrm{e}}$ increase linearly with carrier concentration, and again agree exceptionally with experiments at low carrier concentrations $<10^{19}$ $\mathrm{cm}^{-3}$. However, $s$ and $k_{\mathrm{e}}$ are overestimated at elevated carrier concentration due to the exclusion of extrinsic scattering mechanisms such as defect and boundary scattering in our simulations. The Wiedemann-Franz law connects $\sigma$ and $k_{\mathrm{e}}$ by $k_{\mathrm{e}}=L \sigma T$, where $L$ is the Lorenz number. The Lorenz number ranges between $1.53 \times 10^{-8}$ and $2.38 \times 10^{-8} \mathrm{~W} \Omega \mathrm{K}^{-2}$ when carrier concentration changes from $10^{18} \mathrm{~cm}^{-3}$ to $2 \times 10^{21} \mathrm{~cm}^{-3}$, and compares well with the $\mathrm{Mg}$ doped BiOCuSe at low carrier concentration (Fig. $3 \mathrm{~d}$ ). It becomes closer to the theoretical value for metals $\left(2.44 \times 10^{-8} \mathrm{~W} \Omega \mathrm{K}^{-2}\right)$ at high carrier concentrations. As shown in Fig. 3e, the theoretical limit of the power factor is $21.3 \mu \mathrm{W} \mathrm{cm}{ }^{-1} \mathrm{~K}^{-2}$ at a carrier concentration of $2.0 \times 10^{20} \mathrm{~cm}^{-3}$, which is approximately double the values currently achieved experimentally in various doped systems. The experimental lattice thermal conductivity of polycrystalline BiOCuSe samples (Fig. S1) is used to calculate the thermoelectric figure of merit of BiOCuSe. ${ }^{18}$ The optimal room temperature $Z T$ is calculated to be 0.48 at $1.0 \times 10^{20} \mathrm{~cm}^{-3}$. As shown in Fig. $3 \mathrm{f}$, most of the experimental $Z T$ values are below the theoretical values presented in this study. Some however become larger than the theoretical value at higher carrier concentrations. The possible reason is that microstructural effects, such as creation of nanodots ${ }^{19}$ and all-scale structural optimization ${ }^{21}$ are employed in experiments to reduce the lattice thermal conductivity below the polycrystalline value used here. ${ }^{18}$

\section{Discussion}


Our analysis on the effect of different phonon scattering mechanisms on carrier mobility in BiOCuSe has shown that LA and PO modes dominate charge transport at different temperatures; the PO contributions can be further partitioned into modes from the Cu-Se layer below $200 \mathrm{~cm}^{-1}$ and from the Bi-O layer above $200 \mathrm{~cm}^{-1}$, each becoming the major mobility-limiting mechanism under different conditions. Here we investigate whether the different phonons also show a different effect on power factor and figure of merit $Z T$, by excluding from the calculations the contribution of $P O$ phonons with frequencies $<200 \mathrm{~cm}^{-1}$ and $>200 \mathrm{~cm}^{-1}$, respectively. Figure $3 e$ shows that the optimal power factor can indeed be significantly improved by suppressing specific types of phonons: in particular at low carrier concentrations by suppressing $\mathrm{Cu}-\mathrm{Se}$ phonons, while high carrier concentrations benefit particularly by the suppression of $\mathrm{Bi}-\mathrm{O}$ phonons. The optimal power factor raises to $\sim 30 \mu \mathrm{W} \mathrm{cm}-1 \mathrm{~K}^{-2}$ at the carrier concentration of $8.0 \times$ $10^{19} \mathrm{~cm}^{-3}$ when Cu-Se phonons are excluded. This is mainly due to the increase in electrical conductivity (Fig. S2). A similar increase in the optimal $Z T$ is observed, with maximum of 0.71 at the carrier concentration of $5.0 \times 10^{19} \mathrm{~cm}^{-3}$ when $\mathrm{Cu}$-Se phonons are excluded.

Due to the layered structure and the presence of different anions in each sub-block, BiCuSeO has phonon bands well separated in frequency space that can be engineered independently and optimised to yield best performance under specific $T$ and carrier concentration conditions through targeting specific phonons. In summary, by combining density functional theory calculations with electron-phonon scattering models we demonstrated the fundamental role of electron-PO phonon scattering in charge transport properties of BiOCuSe. An intrinsic hole mobility of $\sim 32 \mathrm{~cm}^{2} \mathrm{~V}^{-1} \mathrm{~s}^{-1}$ at room temperature is predicted under the combined scattering effects of $\mathrm{PO}$ and LA phonons, which compares well to the experimental value of $\sim 20 \mathrm{~cm}^{2} \mathrm{~V}^{-1} \mathrm{~s}^{-1} .{ }^{15}$ The calculated power law of $\mu \sim T^{-1.5}$ also agrees well with experiments. Due to multiple valley effects, the LA phonon limited mobility in BiOCuSe follows a power law of $\mu \sim T^{2.4}$, rather than the commonly assumed $\mu \sim T^{1.5} \cdot{ }^{15,23,24}$ Furthermore, the groups of polar optical phonon modes (four with frequency $<200 \mathrm{~cm}^{-1}$ mainly due to atomic vibrations in the Cu-Se layer and two with frequency $>200 \mathrm{~cm}^{-1}$ localised in the Bi-O layer) have comparable scattering effects between $300-875 \mathrm{~K}$. This explains the experimental observation that improved carrier mobility can be obtained by doping Te into the Cu-Se layer, weakening carrier-phonon interaction. ${ }^{22}$ The theoretical limit $Z T$ of BiOCuSe is calculated to be 2.5 at $875 \mathrm{~K}$. Weakening the coupling between carriers and PO phonons in the Bi-O layer is also a possible strategy for enhancing $Z T$. Our work provides therefore valuable new insight for further rational optimization of charge transport and thermoelectric properties of BiOCuSe through targeting specific phonons. This approach is likely applicable to other materials composed of subunits with distinct characteristic frequencies.

\section{Methods}

The Vienna ab-initio simulation package (VASP) ${ }^{38,39}$ was employed to calculate the structural, vibrational, and electrical properties of BiOCuSe. The optB86b functional ${ }^{40}$ was used to optimize the crystal structure accounting for the van der Waals interactions between layers. The cutoff energy of the plane wave basis 
set was set to $550 \mathrm{eV}$. The k-mesh in optimization was $11 \times 11 \times 5$. The energy and force convergence criteria were $1 \times 10^{-5} \mathrm{eV}$ and $1 \times 10^{-2} \mathrm{eV} / \AA$, respectively. The PBE $+\mathrm{U}+\mathrm{SOC}$ functional (SOC spin-orbit coupling) was employed to calculate the band structure of BiOCuSe. A Hubbard $U$ value of $4 \mathrm{eV}$ was added to the $\mathrm{Cu} 3 \mathrm{~d}$ orbitals, because the $\mathrm{Cu} 3 \mathrm{~d}$ electrons play an important role in Cu based multinary semicondutors. ${ }^{29,41}$ The phonon band structure and phonon density of states (DOS) were obtained by combining VASP with phonopy. ${ }^{42}$ A $4 \times 4 \times 4$ supercell (512 atoms) was built to calculate the phonon band structure. The longitudinal optical and transvers optical (LO-TO) splitting was included. Born effective charges in Table S2.

The Boltzmann transport theory in relaxation time approximation as implemented in the BoltzTraP code ${ }^{43}$ was utilized to calculate the charge carrier mobility and thermoelectric coefficients of BiOCuSe. The electrical conductivity $\sigma$, Seebeck coefficients $S$, electrical thermal conductivity $\kappa_{\mathrm{e}}$, carrier concentration $n$, and charge carrier mobility $\mu$ are calculated as

$$
\begin{aligned}
\sigma & =\frac{e^{2}}{\Omega} \int \tau_{k} v_{k} v_{k}\left(-\frac{\partial f_{0}\left(\varepsilon_{k}\right)}{\partial \varepsilon_{k}}\right) d k \\
S & =\frac{e}{\sigma T} \int \tau_{k} v_{k} v_{k}\left(\varepsilon_{k}-\varepsilon_{f}\right)\left(-\frac{\partial f_{0}\left(\varepsilon_{k}\right)}{\partial \varepsilon_{k}}\right) d k \\
\kappa_{e} & =-S^{2} \sigma T+\frac{1}{\Omega} \int \tau_{k} v_{k} v_{k} \frac{\left(\varepsilon_{k}-\varepsilon_{f}\right)^{2}}{T}\left(-\frac{\partial f_{0}\left(\varepsilon_{k}\right)}{\partial \varepsilon_{k}}\right) d k \\
n & =N-\int g\left(\varepsilon_{k}\right) f_{0}\left(\varepsilon_{k}\right) d k \\
\mu & =\sigma / n e
\end{aligned}
$$

where $\varepsilon_{k}$ is the electron energy, $v_{k}$ is the group velocity, $\tau_{k}$ is the relaxation time, $e$ is the elementary charge, $T$ is the absolute temperature, $\Omega$ is the unit cell volume, $f_{0}$ is the Fermi-Dirac distribution function, $\varepsilon_{f}$ is the Fermi energy, gis the electric density of states, and $N$ is the number of electrons within a unit cell. A fine k-mesh of $31 \times 31 \times 11$ was adopted to calculate the electron energy, which was further interpolated 15 times in BoltzTraP in order to converge the calculation of the above charge transport coefficients.

We implemented relaxation time calculation in BoltzTraP to investigate the LA and PO phonon scattering mechanisms. The total relaxation time is calculated according to Matthiessen's rule 


$$
\frac{1}{\tau(k)}=\frac{1}{\tau_{L A}(k)}+\frac{1}{\tau_{P O}(k)}
$$

The deformation potential theory is used to calculate the LA phonon scattering limited relaxation time by: ${ }^{31}$

$$
\frac{1}{\tau_{L A}(k)}=\sum_{k^{\prime}} \frac{2 \pi k_{B} T E_{1}^{2}}{\hbar c_{i i}} \delta\left(\varepsilon(k)-\varepsilon\left(k^{\prime}\right)\right)(1-\cos \theta)
$$

where $E_{1}$ is the deformation potential constant, $C_{i i}$ is the elastic constant, $k_{B}$ is the Boltzmann constant, $\hbar$ is the reduced Plank constant, and $\theta$ is the scattering angle between $k$ and $k^{\prime}$. The elastic constant $C_{i i}$ was calculated by using DFPT formalism in VASP with optB86b functional. The deformation potential constant E1 was calculated by firstly applying a small strain on the unit cell and then performing a linear fitting of the variation of valence band maximum (VBM) with strain for holes. The strained cells were optimized by using optB86b functional and the band edge energies were calculated by using PBE $+\mathrm{U}+$ SOC functional as the electronic structure is better described by the latter. ${ }^{29}$ The deformation potential constants $E_{1}$ were calibrated by the $\mathrm{Cu} 3 p$ core level energy at the $\Gamma$ point.

The relaxation time due to $\mathrm{PO}$ phonon scattering is calculated by: ${ }^{28,44}$

$$
\begin{aligned}
& \frac{1}{\tau_{P O}(k)}=\frac{e^{2} \omega_{P O}}{4 \sqrt{2} \pi \varepsilon_{0} \hbar}\left(\frac{1}{k_{\infty}}-\frac{1}{k_{0}}\right) \frac{\sqrt{m^{*}}}{\sqrt{E}}\left[n_{q} \frac{f_{0}\left(E-\hbar \omega_{P O}\right)}{f_{0}(E)}\left(\sqrt{1-\frac{\hbar \omega_{P O}}{E}}+\frac{\hbar \omega_{P O}}{E} \cosh ^{-1}\left(\frac{E}{\hbar \omega_{P O}}\right)^{1 / 2}\right)\right. \\
& \left.+\left(n_{q}+1\right) \frac{f_{0}\left(E+\hbar \omega_{P O}\right)}{f_{0}(E)}\left(\sqrt{1+\frac{\hbar \omega_{P O}}{E}}-\frac{\hbar \omega_{P O}}{E} \sinh ^{-1}\left(\frac{E}{\hbar \omega_{P O}}\right)^{1 / 2}\right)\right]
\end{aligned}
$$

when $E>\hbar \omega_{P O}$, and

$$
\frac{1}{\tau_{P O}(k)}=\frac{e^{2} \omega_{P O}}{4 \sqrt{2} \pi \varepsilon_{0} \hbar}\left(\frac{1}{k_{\infty}}-\frac{1}{k_{0}}\right) \frac{\sqrt{m^{*}}}{\sqrt{E}}\left[2\left(n_{q}+1\right) \frac{f_{0}\left(E+\hbar \omega_{P O}\right)}{f_{0}(E)} \sinh ^{-1}\left(\frac{E}{\hbar \omega_{P O}}\right)^{1 / 2}\right]
$$

when $E<\hbar \omega_{\mathrm{PO}}$, where $E$ is the electron energy, $\mathrm{m}^{\star}$ is the effective mass, $\varepsilon_{0}$ is the vacuum permittivity, $\mathrm{k}_{0}$ is the static and $k_{\infty}$ the high-frequency dielectric constant, $\omega_{P O}$ is the frequency of the PO phonon mode, $n_{q}$ is the Bose-Einstein distribution function of phonons. $E$ is calculated with respect to VBM and CBM for holes and electrons, respectively. $m^{\star}$ is obtained by fitting a parabola near the band edges by $E=\left(\hbar^{\wedge} 2 k^{\wedge} 2\right)$ / $\left(2 m^{\wedge *}\right)$. Since usually $k_{0} \gg k_{\infty}, 1 k_{0}$ is omitted. $k_{\infty}$ of BiOCuSe is calculated to be 17.61 and 13.25 for inplane and out of plane directions, respectively, which agree well with previous calculations (18.01 and 
13.79 in Ref. 30; 15.3 and 12.0 in Ref. 45). The relaxation time due to multiple PO phonon modes was also added by using the Matthiessen's rule.

\section{Declarations}

\section{Competing financial interests:}

The authors declare no competing financial interests.

\section{Acknowledgements}

We thank EPSRC for funding under EP/N004884. We thank the UK Materials Chemistry Consortium funded by EPSRC (EP/R029431) for computational resources on ARCHER (http://www.archer.ac.uk) and the UK Materials and Molecular Modelling Hub (EP/P020194) for access to THOMAS.

Author contributions

F.C. and T.Z. proposed the research, T.Z. conducted the calculations. T.Z. prepared all the figures and tables. T.Z. and F.C. analyzed the data and wrote the paper. All the authors commented on the manuscript.

\section{References}

1. Witting, I. T. et al. The Thermoelectric Properties of Bismuth Telluride. Adv. Electron. Mater. 5, 1800904 (2019).

2. Kütt, L., Millar, J., Karttunen, A., Lehtonen, M. \& Karppinen, M. Thermoelectric applications for energy harvesting in domestic applications and micro-production units. Part I: Thermoelectric concepts, domestic boilers and biomass stoves. Renew. Sustain. Energy Rev. 98, 519-544 (2018).

3. He, J., Liu, Y. \& Funahashi, R. Oxide thermoelectrics: The challenges, progress, and outlook. J. Mater. Res. 26, 1762-1772 (2011).

4. Ohtaki, M. Recent aspects of oxide thermoelectric materials for power generation from mid-to-high temperature heat source. J. Ceram. Soc. Japan 119, 770-775 (2011).

5. Wu, T. \& Gao, P. Development of perovskite-type materials for thermoelectric application. Materials (Basel). 11, 999 (2018).

6. Terasaki, I., Sasago, Y. \& Uchinokura, K. Large thermoelectric power in $\mathrm{NaCo}_{2} \mathrm{O}_{4}$ single crystals. Phys. Rev. B 56, R12685-R12687 (1997).

7. Okuda, T., Nakanishi, K., Miyasaka, S. \& Tokura, Y. Large thermoelectric response of metallic perovskites: $\mathrm{Sr}_{1}{ }_{x} \mathrm{La}_{x} \mathrm{TiO}_{3}(0 \leq x \leq 0.1)$. Phys. Rev. B - Condens. Matter Mater. Phys. 63, 113104 (2001). 
8. Liu, R. et al. BiCuSeO as state-of-the-art thermoelectric materials for energy conversion: from thin films to bulks. Rare Met. 37, 259-273 (2018).

9. Zhao, L. D. et al. $\mathrm{Bi}_{1}-{ }_{x} \mathrm{Sr}_{x} \mathrm{CuSeO}$ oxyselenides as promising thermoelectric materials. Appl. Phys. Lett. 97, 092118 (2010).

10. Li, F. et al. Polycrystalline BiCuSeO oxide as a potential thermoelectric material. Energy Environ. Sci. 5, 7188-7195 (2012).

11. Li, J. et al. The roles of $\mathrm{Na}$ doping in BiCuSeO oxyselenides as a thermoelectric material. J. Mater. Chem. A 2, 4903-4906 (2014).

12. Sun Lee, D. et al. Density of state effective mass and related charge transport properties in K-doped BiCuOSe. Appl. Phys. Lett. 103, 232110 (2013).

13. Li, J. et al. Thermoelectric properties of Mg doped p-type BiCuSeO oxyselenides. J. Alloys Compd. 551, 649-653 (2013).

14. Li, F., Wei, T. R., Kang, F. \& Li, J. F. Enhanced thermoelectric performance of Ca-doped BiCuSeO in a wide temperature range. J. Mater. Chem. A 1, 11942-11949 (2013).

15. Barreteau, C., Bérardan, D., Amzallag, E., Zhao, L. D. \& Dragoe, N. Structural and electronic transport properties in Sr-doped BiCuSeO. Chem. Mater. 24, 3168-3178 (2012).

16. $\mathrm{Li}$, J. et al. A high thermoelectric figure of merit $\mathrm{ZT}>1$ in $\mathrm{Ba}$ heavily doped BiCuSeO oxyselenides. Energy Environ. Sci. 5, 8543-8547 (2012).

17. Pan, L., Bérardan, D., Zhao, L., Barreteau, C. \& Dragoe, N. Influence of Pb doping on the electrical transport properties of BiCuSeO. Appl. Phys. Lett. 102, 023902 (2013).

18. Ren, G. K. et al. Enhanced thermoelectric properties in $\mathrm{Pb}$-doped BiCuSeO oxyselenides prepared by ultrafast synthesis. RSC Adv. 5, 69878-69885 (2015).

19. Lan, J. Le et al. Enhanced thermoelectric properties of Pb-doped BiCuSeO ceramics. Adv. Mater. 25, 5086-5090 (2013).

20. Ren, G.-K. et al. Complex electronic structure and compositing effect in high performance thermoelectric BiCuSeO. Nat. Commun. 10, 2814 (2019).

21. Liu, Y. et al. Synergistically Optimizing Electrical and Thermal Transport Properties of BiCuSeO via a Dual-Doping Approach. Adv. Energy Mater. 6, 1502423 (2016).

22. Ren, G.-K. et al. Enhancing thermoelectric performance in hierarchically structured $\mathrm{BiCuSeO}$ by increasing bond covalency and weakening carrier-phonon coupling. Energy Environ. Sci. 10, 15901599 (2017).

23. Fan, D. D. et al. Understanding the electronic and phonon transport properties of a thermoelectric material BiCuSeO: a first-principles study. Phys. Chem. Chem. Phys. 19, 12913-12920 (2017).

24. Lan, J.-L. et al. Doping for higher thermoelectric properties in p-type BiCuSeO oxyselenide. Appl. Phys. Lett. 102, 123905 (2013).

25. Liu, J., Jiang, Q., Zhang, S. \& Zhang, H. Carrier mobility and relaxation time in BiCuSeO. Phys. Lett. A 1,125990 (2019). 
26. Zhou, J. J., Hellman, O. \& Bernardi, M. Electron-Phonon Scattering in the Presence of Soft Modes and Electron Mobility in $\mathrm{SrTiO}_{3}$ Perovskite from First Principles. Phys. Rev. Lett. 121, 226603 (2018).

27. Himmetoglu, B., Janotti, A., Peelaers, H., Alkauskas, A. \& Van de Walle, C. G. First-principles study of the mobility of $\mathrm{SrTiO}_{3}$. Phys. Rev. B 90, 241204 (2014).

28. Filippetti, A., Mattoni, A., Caddeo, C., Saba, M. I. \& Delugas, P. Low electron-polar optical phonon scattering as a fundamental aspect of carrier mobility in methylammonium lead halide $\mathrm{CH}_{3} \mathrm{NH}_{3} \mathrm{Pbl}_{3}$ perovskites. Phys. Chem. Chem. Phys. 18, 15352-15362 (2016).

29. Gibson, Q. D. et al. $\mathrm{Bi}_{2+2 n} \mathrm{O}_{2+2 n} \mathrm{Cu}_{2-\delta} \mathrm{Se}_{2+n-\delta} \mathrm{X}_{\delta}(\mathrm{X}=\mathrm{Cl}, \mathrm{Br})$ : A Three-Anion Homologous Series. Inorg. Chem. 57, 12489-12500 (2018).

30. Saha, S. K. Exploring the origin of ultralow thermal conductivity in layered BiOCuSe. Phys. Rev. B 92, 041202 (2015).

31. Xi, J., Long, M., Tang, L., Wang, D. \& Shuai, Z. First-principles prediction of charge mobility in carbon and organic nanomaterials. Nanoscale 4, 4348-4369 (2012).

32. Li, Y. H., Gong, X. G. \& Wei, S. H. Ab initio all-electron calculation of absolute volume deformation potentials of IV-IV, III-V, and II-VI semiconductors: The chemical trends. Phys. Rev. B - Condens. Matter Mater. Phys. 73, 245206 (2006).

33. Zhao, T., Shi, W., Xi, J., Wang, D. \& Shuai, Z. Intrinsic and Extrinsic Charge Transport in $\mathrm{CH}_{3} \mathrm{NH}_{3} \mathrm{Pbl}$ Perovskites Predicted from First-Principles. Sci. Rep. 6, 19968 (2016).

34. Mante, P.-A., Stoumpos, C. C., Kanatzidis, M. G. \& Yartsev, A. Electron-acoustic phonon coupling in single crystal $\mathrm{CH}_{3} \mathrm{NH}_{3} \mathrm{Pbl}_{3}$ perovskites revealed by coherent acoustic phonons. Nat. Commun. 8 , 14398 (2017).

35. Pei, Y.-L. et al. High thermoelectric performance of oxyselenides: intrinsically low thermal conductivity of Ca-doped BiCuSeO. NPG Asia Mater. 5, e47 (2013).

36. Zhu, H. et al. Promoted high temperature carrier mobility and thermoelectric performance of InTe enabled by altering scattering mechanism. J. Mater. Chem. A 7, 11690-11698 (2019).

37. Mahan, G. D. \& Sofo, J. O. The best thermoelectric. Proc. Natl. Acad. Sci. 93, 7436-7439 (1996).

38. Kresse, G. \& Furthmüller, J. Efficiency of ab-initio total energy calculations for metals and semiconductors using a plane-wave basis set. Comput. Mater. Sci. 6, 15-50 (1996).

39. Kresse, G. \& Furthmüller, J. Efficient iterative schemes for ab initio total-energy calculations using a plane-wave basis set. Phys. Rev. B - Condens. Matter Mater. Phys. 54, 11169-11186 (1996).

40. Klime, J., Bowler, D. R. \& Michaelides, A. Van der Waals density functionals applied to solids. Phys. Rev. B - Condens. Matter Mater. Phys. 83, 195131 (2011).

41. Zhang, Y. et al. Comparative study of structural and electronic properties of Cu-based multinary semiconductors. Phys. Rev. B - Condens. Matter Mater. Phys. 84, 075127 (2011).

42. Togo, A. \& Tanaka, I. First principles phonon calculations in materials science. Scr. Mater. 108, 1-5 (2015). 
43. Madsen, G. K. H. \& Singh, D. J. BoltzTraP. A code for calculating band-structure dependent quantities. Comput. Phys. Commun. 175, 67-71 (2006).

44. Ridley, B. K., Tripathi, P., Ridley, B. K. \& Ridley, B. K. Polar-optical-phonon and electron-electron scattering in large-bandgap semiconductors. J. Phys.: Condens. Matter 10, 6717-6726 (1998).

45. Kumar, S. \& Schwingenschlögl, U. Lattice thermal conductivity in layered BiCuSeO. Phys. Chem. Chem. Phys. 18, 19158-19164 (2016).

\section{Figures}

(a)

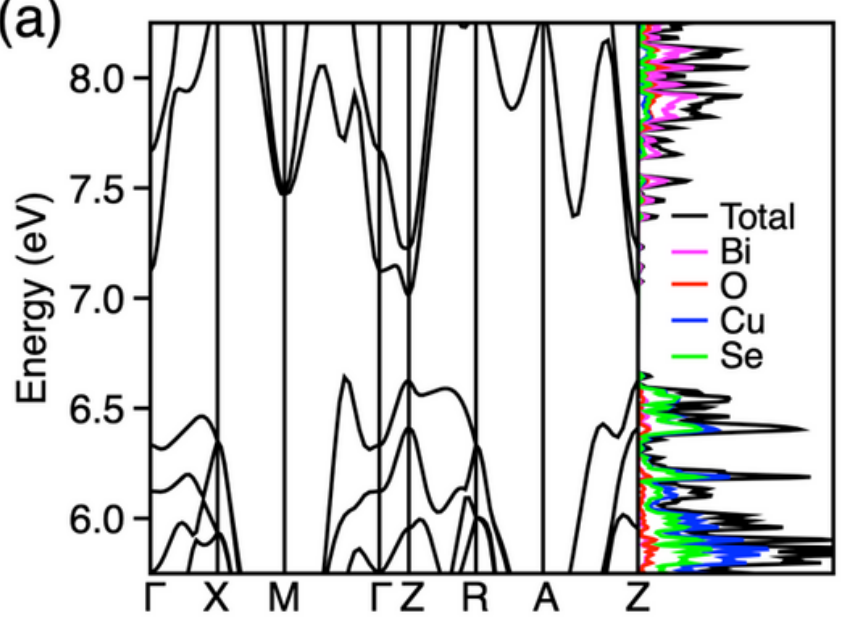

(b)

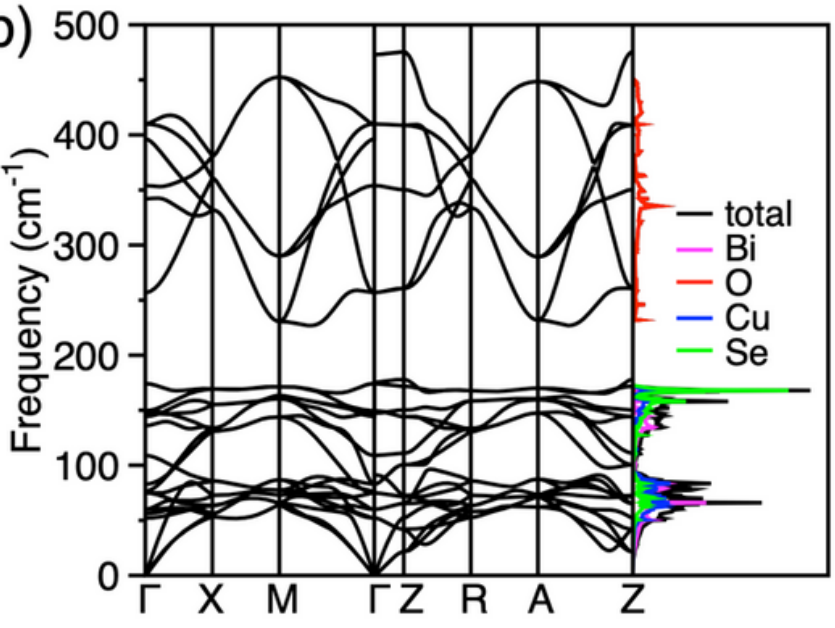

(c)

(d)
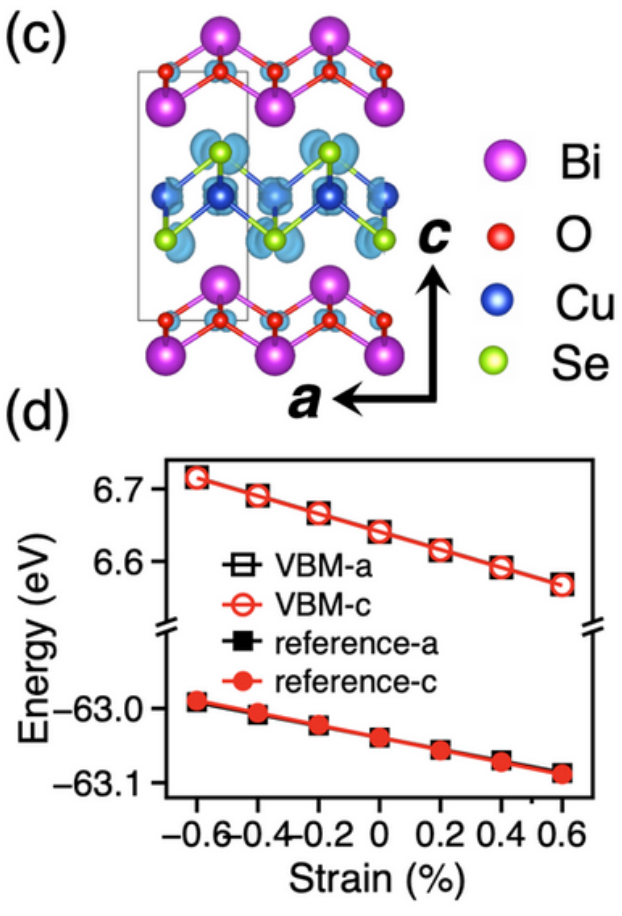

(e) $E_{u} 56.2 \mathrm{~cm}^{-1}$

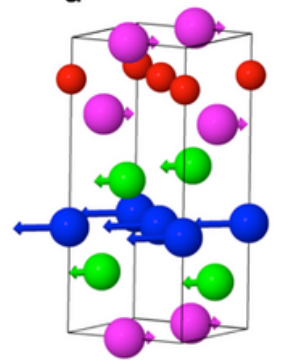

$A_{2 u} 175.2 \mathrm{~cm}^{-1}$

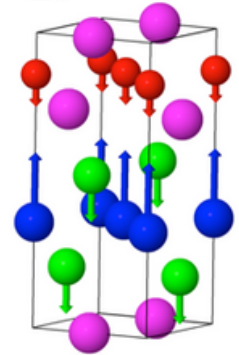

$A_{2 u} 91.4 \mathrm{~cm}^{-1}$

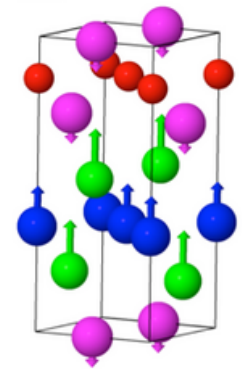

$E_{u} 341.9 \mathrm{~cm}^{-1}$

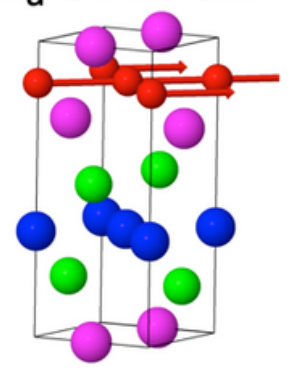

$E_{\mathrm{u}} 136.0 \mathrm{~cm}^{-1}$

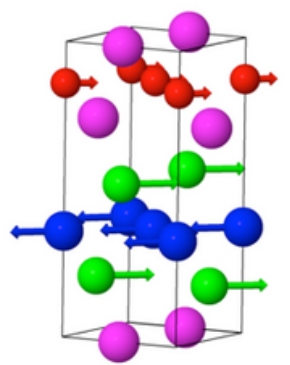

$A_{2 u} 472.9 \mathrm{~cm}^{-1}$

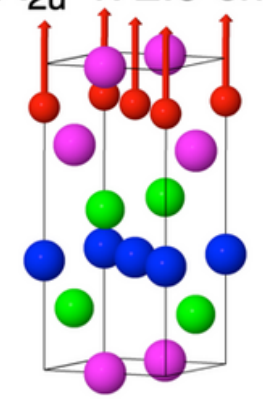

Figure 1

(a) Electronic band structure and density of states (DOS) of BiOCuSe. (b) Phonon band structure and density of states. (c) Crystal structure of BiOCuSe, and charge distribution for the eigenstates at the VBM. 
(d) Variation of VBM energy with strain in a and c directions. The reference energy of the Cu-3p core level used to obtain the deformation constants is also shown. (e) Symmetry, frequency and atomic displacements for the PO phonon modes at $\Gamma$.
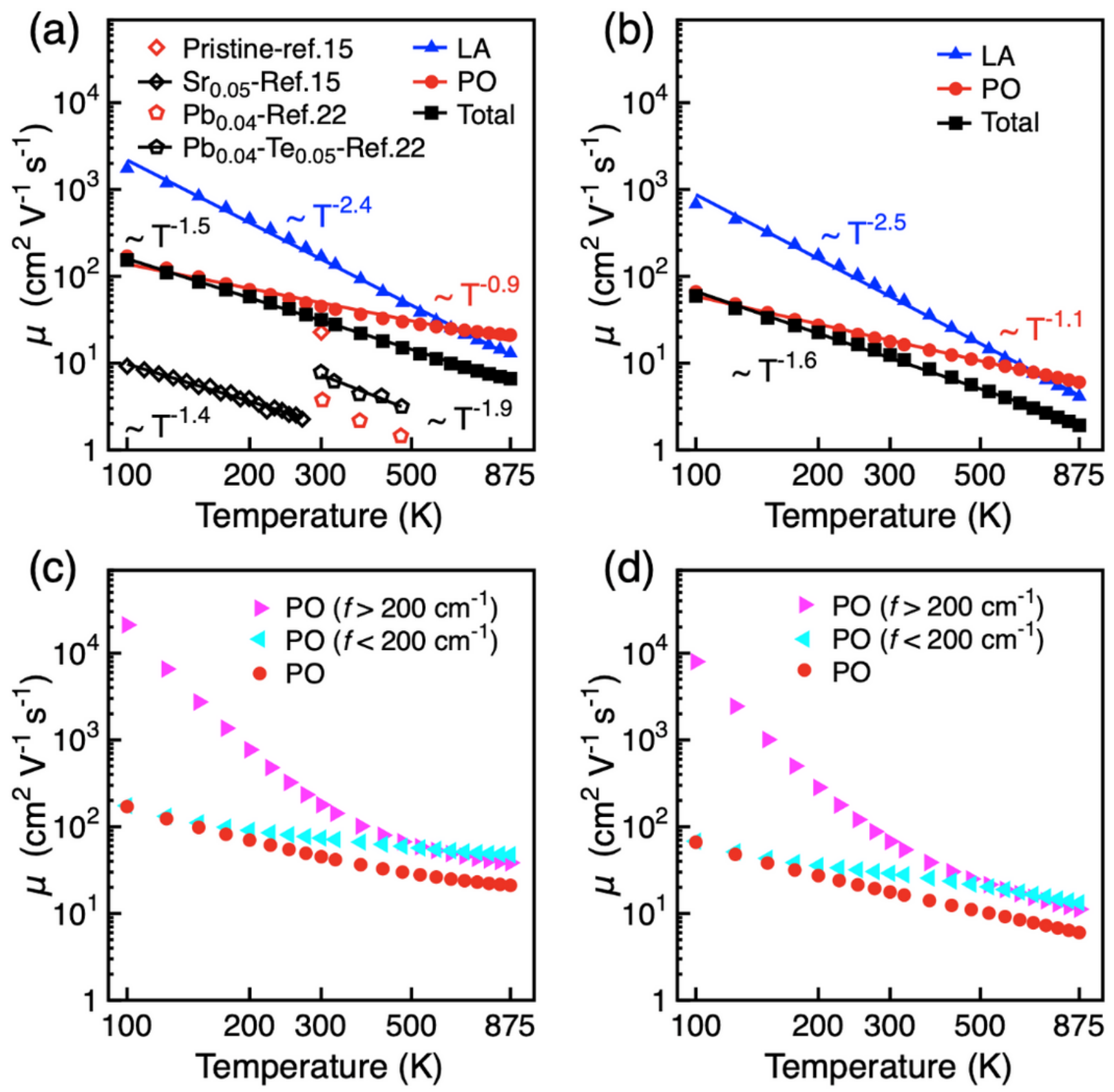

Figure 2

Hole mobility $\mu$ of BiOCuSe as a function of temperature: LA, PO, and total scattering limited mobility in (a) a direction and (b) c direction. Experimental results are shown for comparison. Hole mobility calculated by considering PO scattering only, and separate contributions from the PO modes with frequency $>200 \mathrm{~cm}-1$ and $<200 \mathrm{~cm}-1$, respectively, in (c) a direction and (d) c direction. 


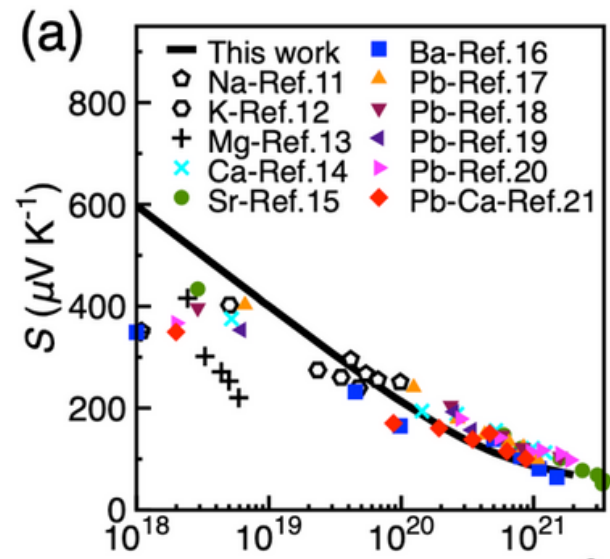

Carrier Concentration $\left(\mathrm{cm}^{-3}\right)$

(d)

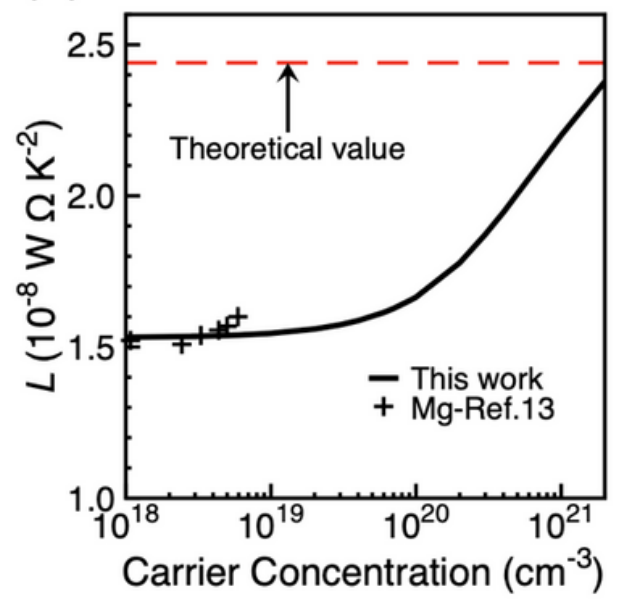

(b)

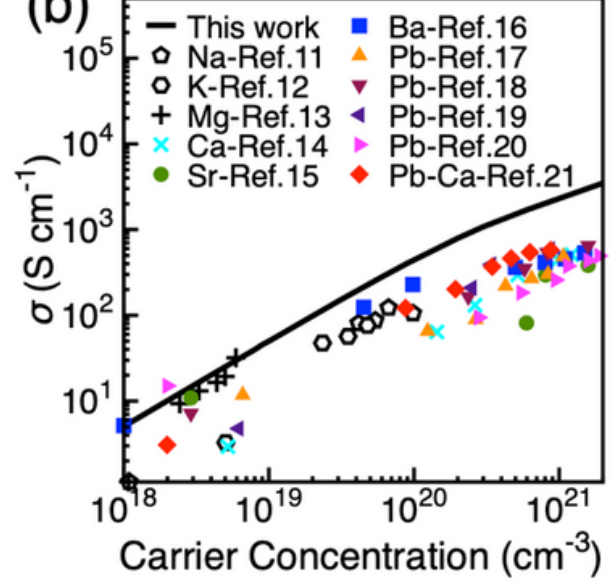

(e)

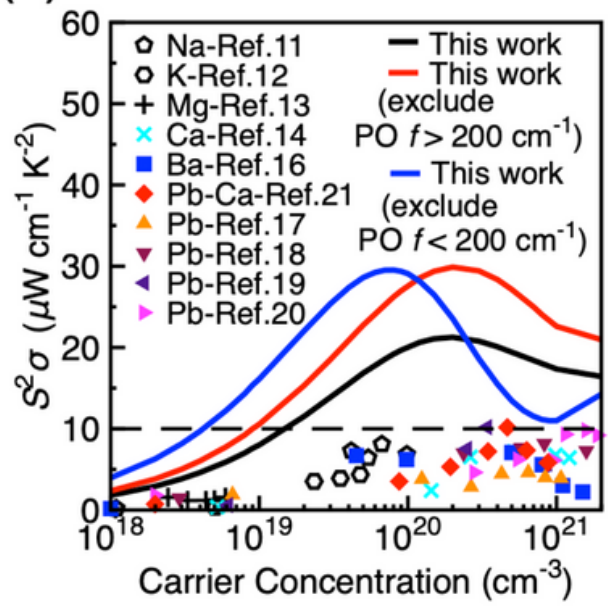

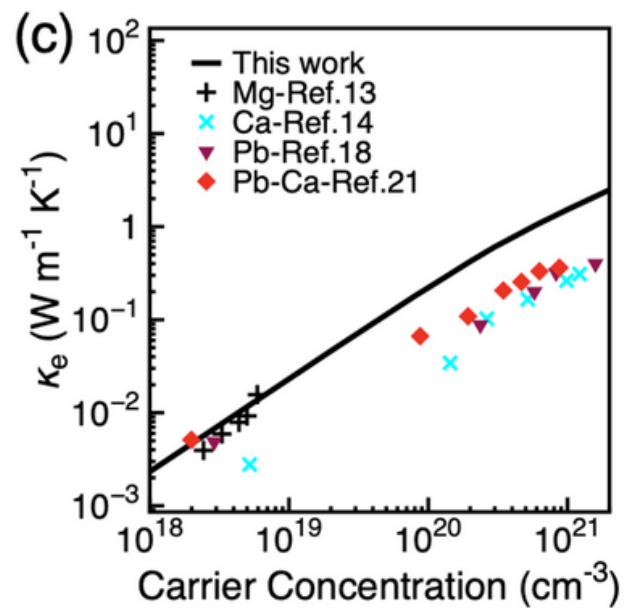

(f)

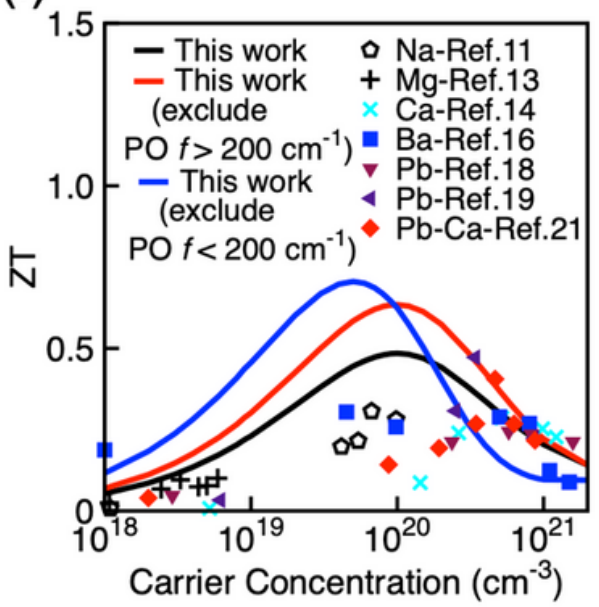

Figure 3

Thermoelectric observables as a function of carrier concentration: (a) Seebeck coefficient S, (b) electrical conductivity $\sigma$, (c) electrical thermal conductivity ke, (d) Lorenz number $L$, (e) thermoelectric power factor $S 2 \sigma$, and (f) thermoelectric figure of merit ZT. Experimental values under various doping methods are shown for comparison. 

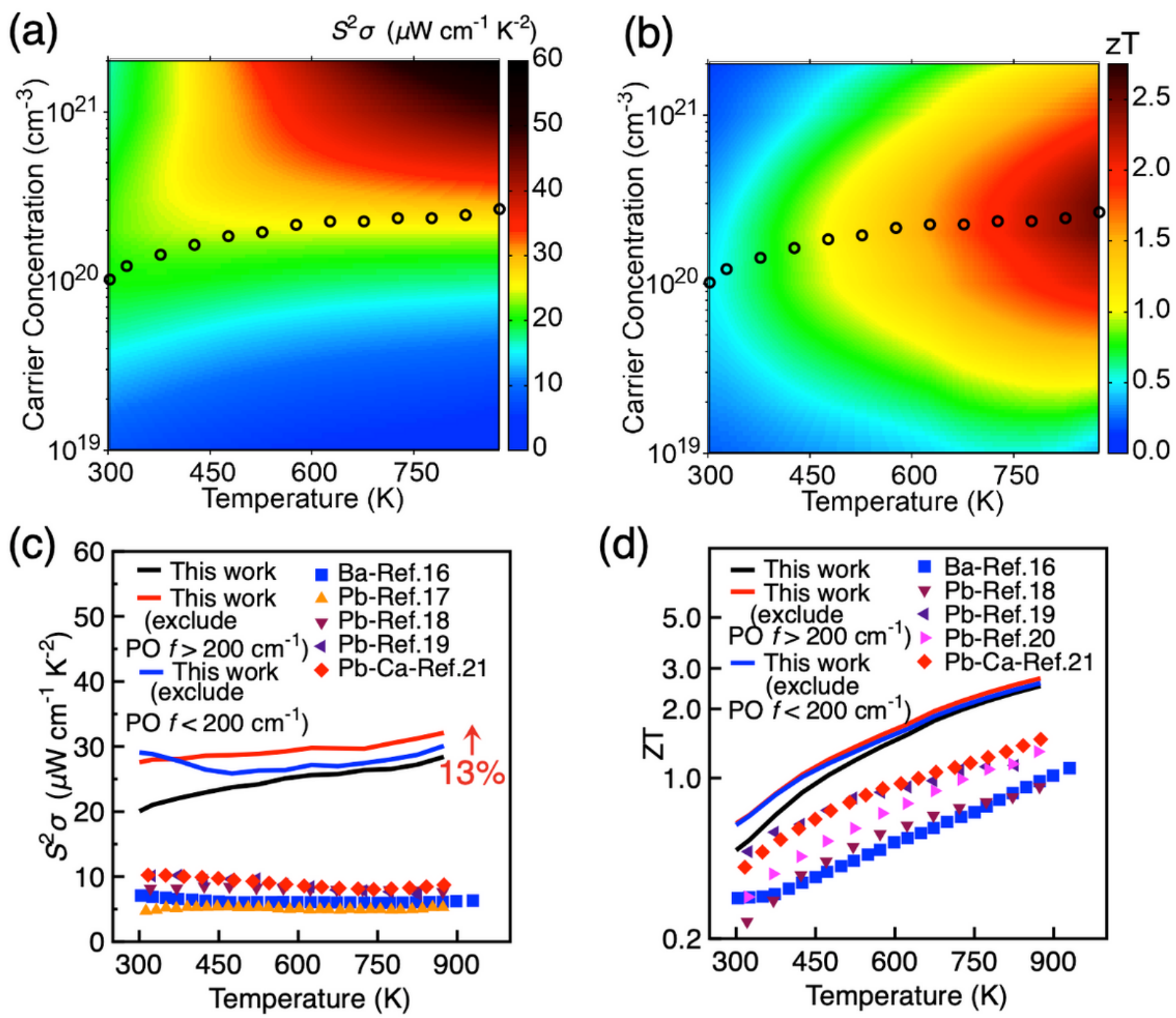

(d)

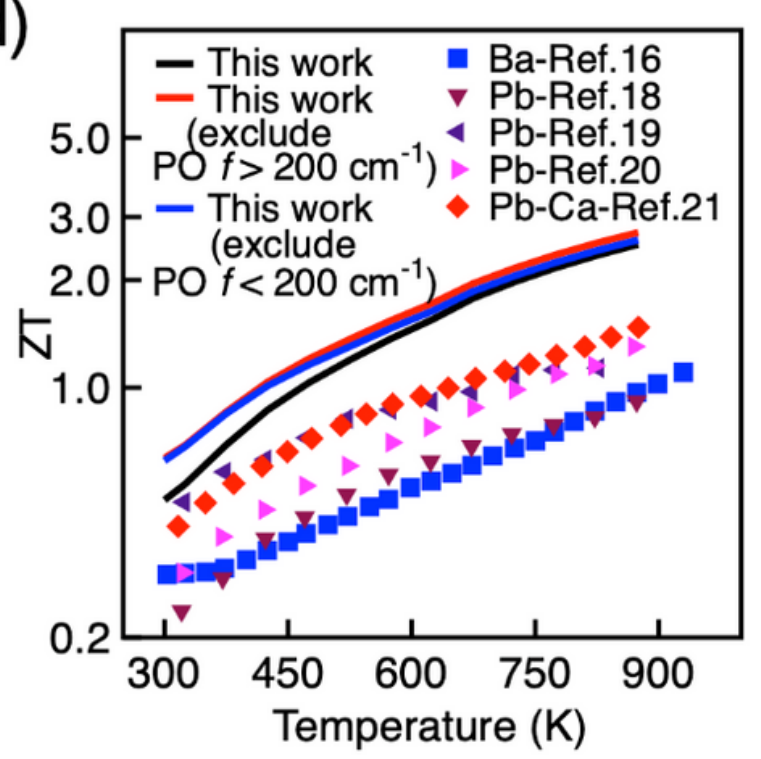

Figure 4

(a) Variation of power factor $S 2 \sigma$ and (b) thermoelectric figure of merit ZT with temperature and carrier concentration for p-type BiOCuSe. The circles denote the position of maximum ZT at each temperature. (c) Power factor and (d) optimal figure of merit ZT in the temperature range of 300-875 K. Experimental values under various doping methods are shown for comparison.

\section{Supplementary Files}

This is a list of supplementary files associated with this preprint. Click to download.

- Supplementary.docx 